\title{
Clinical features and prognostic factors of intensive and non-intensive 1014 COVID-19 patients: an experience cohort from Alahsa, Saudi Arabia
}

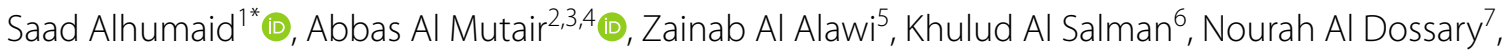
Ahmed Omar ${ }^{8}$, Mossa Alismail ${ }^{9}$, Ali M. Al Ghazal ${ }^{10}$, Mahdi Bu Jubarah ${ }^{9}$, Hanan Al Shaikh ${ }^{9}$, Maher M. Al Mahdi ${ }^{10}$, Sarah Y. Alsabati ${ }^{11}$, Dayas K. Philip ${ }^{12}$, Mohammed Y. Alyousef ${ }^{13}$, Abdulsatar H. Al Brahim ${ }^{14}$, Maitham S. Al Athan ${ }^{15}$, Salamah A. Alomran ${ }^{14}$, Hatim S. Ahmed ${ }^{15}$, Haifa Al-Shammari ${ }^{16}$, Alyaa Elhazmi ${ }^{17}$, Ali A. Rabaan ${ }^{18}$, Jaffar A. Al-Tawfiq ${ }^{19,20,21}$ and Awad Al-Omari'22,23

\begin{abstract}
Background: COVID-19 is a worldwide pandemic and has placed significant demand for acute and critical care services on hospitals in many countries.

Objectives: To determine the predictors of severe COVID-19 disease requiring admission to an ICU by comparing patients who were ICU admitted to non-ICU groups.

Methods: A cohort study was conducted for the laboratory-confirmed COVID-19 patients who were admitted to six Saudi Ministry of Health's hospitals in Alahsa, between March 1, 2020, and July 30, 2020, by reviewing patient's medical records retrospectively.

Results: This cohort included 1014 patients with an overall mean age of $47.2 \pm 19.3$ years and 582 (57\%) were males. A total of 205 (20\%) of the hospitalized patients were admitted to the ICU. Hypertension, diabetes and obesity were the most common comorbidities in all study patients $(27.2,19.9$, and $9 \%$, respectively). The most prevalent symptoms were cough (47.7\%), shortness of breath (35.7\%) and fever (34.3\%). Compared with non-ICU group, ICU patients had older age $(p \leq 0.0005)$ and comprised a higher proportion of the current smokers and had higher respiratory rates $(p \leq 0.0005)$, and more percentage of body temperatures in the range of $37.3-38.0^{\circ} \mathrm{C}(p \geq 0.0005)$; and had more comorbidities including diabetes ( $p \leq 0.0005)$, hypertension ( $p \geq 0.0005)$, obesity $(p=0.048)$, and sickle cell disease $(p=0.039)$. There were significant differences between the non-ICU and ICU groups for fever, shortness of breath, cough, fatigue, vomiting, dizziness; elevated white blood cells, neutrophils, alanine aminotransferase and alkaline aminotransferase, lactate dehydrogenase, and ferritin, and decreased hemoglobin; and proportion of abnormal bilateral chest CT images $(p<0.05)$. Significant differences were also found for multiple treatments $(p<0.05)$. ICU patients group had a much higher mortality rate than those with non-ICU admission ( $p \leq 0.0005)$.
\end{abstract}

\footnotetext{
*Correspondence: saalhumaid@moh.gov.sa

${ }^{1}$ Administration of Pharmaceutical Care, Alahsa Health Cluster, Ministry of Health, Rashdiah Street, P. O. Box 12944, Alahsa 31982, Saudi Arabia

Full list of author information is available at the end of the article
}

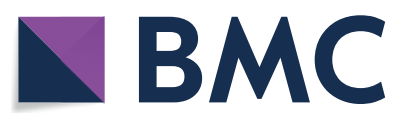

(c) The Author(s) 2021. This article is licensed under a Creative Commons Attribution 4.0 International License, which permits use, sharing, adaptation, distribution and reproduction in any medium or format, as long as you give appropriate credit to the original author(s) and the source, provide a link to the Creative Commons licence, and indicate if changes were made. The images or other third party material in this article are included in the article's Creative Commons licence, unless indicated otherwise in a credit line to the material. If material is not included in the article's Creative Commons licence and your intended use is not permitted by statutory regulation or exceeds the permitted use, you will need to obtain permission directly from the copyright holder. To view a copy of this licence, visit http://creativeco mmons.org/licenses/by/4.0/. The Creative Commons Public Domain Dedication waiver (http://creativecommons.org/publicdomain/ zero/1.0/) applies to the data made available in this article, unless otherwise stated in a credit line to the data. 
Conclusion: Identifying key clinical characteristics of COVID-19 that predict ICU admission and high mortality can be useful for frontline healthcare providers in making the right clinical decision under time-sensitive and resourceconstricted environment.

Keywords: Admission, Clinical, Characteristics, COVID-19, ICU, Intensive, Care, Critical, Mortality, Outcomes, SARSCoV-2, Saudi Arabia

\section{Background}

A novel coronavirus was recognized as the cause of a cluster of pneumonia cases before the end of 2019 and spread throughout China and elsewhere [1]. World Health Organization (WHO) has officially named the virus severe acute respiratory syndrome coronavirus 2 (SARS-CoV-2) and the disease corona virus disease 2019 (COVID-19) [2]. On January 30, 2020, the WHO declared the COVID-19 outbreak a public health emergency of international concern and, in March 2020, began to characterize it as a pandemic, in order to highlight the seriousness of the situation and urge all countries to take action in detecting infection and preventing spread. The main transmission route of SARS-CoV-2 is through the air (airborne transmission) and on a daily basis the number of deaths associated with COVID-19 is rapidly increasing [3]. Many countries have started the safety measures and precautions through screening people coming from overseas including China. Saudi Arabia has started early its precautions and implemented screening for travelers. A governmental move followed by several decisions made to prevent and control the outbreak. The Saudi Ministry of Health $(\mathrm{MoH})$ and the Center for Disease Prevention and Control in the Kingdom have published the Coronavirus Infection Guidelines [4] along with other guidelines that should help to detect, prevent and manage the epidemic. On March 02, 2020, Saudi Arabia reported the first confirmed case of COVID-19 infection in an adult Saudi national who returned from Iran via Bahrain [5]. As of January 5, 2021, the total reported confirmed COVID-19 cases have reached more than 363,259 including more than 6,265 deaths within Saudi Arabia [6]. Studies have shown that up to $20 \%$ of the patients infected with SARS-CoV-2 develop high disease severity and need to be hospitalized [7-9]. Intensive care unit (ICU) admission is a requirement for up to $25 \%$ among those who are hospitalized [8, 10-15]. Patients infected by COVID-19 have a very broad spectrum of illness, from patients being asymptomatic to severally ill requiring an ICU bed admission [16, 17]. Thus, it is crucial to identify COVID-19 patients at risk to develop severe illness, to decrease mortality rate among those patients and improve their clinical outcomes. This report is a cohort study from six Ministry of Health's hospitals in Alahsa Governorate, Saudi Arabia. The study describes the clinical characteristics, laboratories features as well as clinical outcomes for ICU and non-ICU hospitalized COVID-19 patients.

\section{Objectives}

To determine the predictors of severe COVID-19 disease requiring an ICU admission by comparing patients who were ICU admitted to non-ICU groups.

\section{Methods \\ Design}

A cohort study was conducted for the laboratory-confirmed COVID-19 patients who were admitted to six Saudi Ministry of Health's hospitals in Alahsa, namely, the King Fahad Hofuf Hospital, Prince Saud Bin Jalawi Hospital, Maternity and Children Hospital, King Faisal General Hospital, Alomran General Hospital and Aljaber Eye and ENT Hospital, between March 1, 2020, and July 30,2020 , by reviewing patient's medical records retrospectively.

\section{Definitions and ICU eligibility}

SARS-Cov-2 infection, defined as presenting with a fever or any respiratory symptoms, including dry cough, and especially in those with a history of travel or exposure to infected people within 2 weeks before the onset of illness since January 2020. Case definitions of confirmed human infection with SARS-Cov-2 were in accordance with the interim guidance from the WHO [18]. Patients were considered to have the symptom of fever when they had a body temperature $\geq 37.3^{\circ} \mathrm{C}\left(\geq 37.3^{\circ} \mathrm{C}\right.$ orally or $\geq 37.7^{\circ} \mathrm{C}$ rectally) or an elevation above a person's known normal daily value [19].

Guidelines of the Saudi MoH on ICU Triage, Admission, and Discharge Criteria during the COVID 19 pandemic V2 [20] were used to help prepare and plan provision of ICU healthcare for patients included in this study during the ongoing pandemic.

Criteria for ICU admission of COVID-19 patients were [20]:

a. Patient requiring invasive mechanical ventilation

b. Patient requiring more than $2 \mathrm{~h}$ on non-invasive ventilation (NIV) or high-flow nasal cannula (HFNC)

c. Respiratory distress 
(i) Need $\mathrm{O}_{2}>6 \mathrm{~L}$ per minute to maintain $\mathrm{SpO}_{2}>92$ or $\mathrm{PaO}_{2}>65$

(ii) Rapid escalation of oxygen requirement

(iii) Significant work of breathing, i.e., tachypnea

d. Patient with hemodynamic instability despite initial conservative fluid resuscitation

e. Patient requiring vasopressor support

f. Patient with a decreased level of consciousness

g. Acidosis: $\mathrm{ABG}$ with $\mathrm{pH}<7.3$ or $\mathrm{PCO}_{2}>50$ or above patient's baseline. Lactate $>2$

h. Patient with more than one organ failure

i. Patient requires continuous renal preplacement therapy (CRRT) and cannot tolerate hemodialysis

j. Patient with unstable vital signs not yet on vasopressors

k. Patent with new ECG findings, including ischemia, arrhythmias, heart block

Only patients with a laboratory-confirmed infection were enrolled in this study. No exclusion criteria were applied for all confirmed SARS-CoV-2 cases in this study.

\section{Healthcare system of the Alahsa city and setting}

Alahsa Governorate in the Alahsa oasis region in Eastern Saudi Arabia is the largest governorate in the Eastern Province. This region governs both urban and rural populations totaling 1.3 million people. Ministry of Health is the main public healthcare sector that provides preventive, curative, and rehabilitative healthcare services for the entire population of the Alahsa region. When a report of a suspected case of COVID-19 is generated at a primary healthcare center or a medical facility, the patient is referred to a general, specialized, secondary or tertiary care hospital and the relevant health directorates of Ministry of Health are notified. King Fahad Hofuf Hospital is a 500-bed general hospital in Hofuf. It is the biggest hospital in the city area in Alahsa. Other large referral general, specialized, secondary and/or tertiary care hospitals in Alahsa health included in this study were: Maternity and Children Hospital has a 450bed capacity; Prince Saud Bin Jalawi Hospital, a 250-bed capacity facility; both King Faisal General Hospital and Alomran General Hospital are 200-bed capacity facilities; while Prince Sultan Cardiac Center has a capacity of 100 beds. These hospitals provide a vast number of services in several specialties and subspecialties (adult, pediatric and neonatal, cardiology, oncology, internal medicine, infectious diseases, dermatology, gastroenterology, rheumatology, hematology, radiology, geriatrics, obstetrics and gynecology, neuroscience, nephrology, orthopedics, urology, surgery, ear, nose and throat care, dental, burn and intensive care). The hospital records of patients with laboratory-confirmed SARS-Cov-2 infection treated at these hospitals between March 1, 2020, and July 30, 2020, were reviewed retrospectively.

\section{Main outcome measures}

A Microsoft Excel data sheet listing the demographic, clinical, radiography and laboratory variables, and treatment outcomes, were used for the data collection. Variables included patients' information (i.e., case identifying number, sex, age, nationality, level of education, occupation, use of tobacco and exposure history), information on the name of the hospital, data on time from exposure to the onset of illness, and time from illness onset to first hospital admission, patient's medical history and/ or co-morbid conditions, signs and symptoms of SARS$\mathrm{CoV}-2$ illness, time of symptom onset, clinical symptoms, laboratory abnormalities, chest CT findings, medications administered to the patient and treatment outcomes (i.e., hospitalization, discharged transferred or died). Information sources were medical files, electronic health information records and laboratories reports of COVID-19 patients. Patients were stratified based on ICU admission status.

\section{Data management and analysis}

Descriptive statistics were used to describe the data. For categorical variables, frequencies and percentages were reported. Differences between groups were analyzed using the Chi-square $\left(\chi^{2}\right)$ tests (or Fisher's exact tests for expected cell count $<5$ in more than $20 \%$ of the cells). For continuous variables, mean and standard deviation were used to summarize the data and analyses were performed using Student's $t$-tests (Mann-Whitney U test if data are not normally distributed). An a priori two-tailed level of significance was set at 0.05 . Statistical analyses were performed using Microsoft Excel 2010 (Microsoft Corp., Redmond, USA) and IBM SPSS Statistics software, version 22.0 (IBM Corp., Armonk, NY, USA).

\section{Ethics considerations}

This study obtained approval from the Institutional Review Board in King Fahad Hofuf Hospital in Alahsa Health Cluster [IRB KFHH No. (H-05-HS-065)], and was performed according to the Helsinki Declaration. Throughout the study unique patient codes to each study participant were issued to maintain anonymity and confidentiality.

\section{Results}

Demographic and clinical characteristics

The study population included 1,014 hospitalized patients with confirmed COVID-19. The overall mean age of the hospitalized SARS-CoV-2 cohort was $47.2 \pm 19.3$ years, 
ranging from 1 month to $\geq 90$ years. A total of $57.4 \%$ $(n=582)$ of the patients were males and $88.8 \%(n=900)$ were Saudi citizens. About $52.2 \%(n=529)$ of the patients received an educational level of primary to secondary and $2.1 \%(n=21)$ were healthcare providers. Almost 66 percent $(n=668)$ had contact with positive confirmed SARS-CoV-2 cases and $17.8 \%(n=180)$ had familiar or cluster infections. Only one patient had a history of travel to a country with a high risk of COVID-19 transmission. The remaining percentage of the patients $(14.1 \% ; n=144)$ had unknown mode of infection. Hypertension, diabetes and obesity $\left(\mathrm{BMI} \geq 30 \mathrm{~kg} / \mathrm{m}^{2}\right)$ were the most common comorbidities in all study patients $(27.2,19.9$, and 9\%, respectively) (Table 1). As shown in Fig. 1, the most prevalent symptoms at onset of illness were cough $(47.7 \%$; $n=484)$, shortness of breath $(35.7 \% ; n=362)$ and fever $(34.3 \%$; $n=348)$. Minor symptoms were headache $(6.9 \%$; $n=70)$; loss of taste or smell $(5.3 \%$; $n=54)$; gastrointestinal symptoms [diarrhea $(6.9 \% ; n=70)$, vomiting $(2.8 \%$; $n=28)$, abdominal pain $(2.5 \% ; n=25)$, and nausea (1.9\%; $n=19)]$; anorexia $(3.8 \% ; n=39)$; and rash $(1.1 \% ; n=12)$.

A total of $205(20.2 \%)$ of the hospitalized patients were admitted to ICU. As illustrated in Table 1, those patients admitted to the ICU were more likely to have been older in age (45.3 vs 52.9 years; $p \leq 0.0005$ ); tenfold more likely to be current smokers compared to the non-ICU group (2.1 vs $20.5 \%$ ); and suffered the highest body temperatures more in the range of $37.3-38.0{ }^{\circ} \mathrm{C}$; $p \leq 0.0005$. Respiratory rate of $>24$ breaths per minute (bpm) was a lot more common in the ICU patients (20 vs $70 \% ; p \leq 0.0005)$. Comorbidities of hypertension, diabetes and obesity were statistically significant between the two groups (hypertension: $20.4 \%$ for non-ICU group vs $54.1 \%$ for ICU group, $p \leq 0.0005$; diabetes: $17.5 \%$ for non-ICU group vs $29.3 \%$ for ICU group, $p \leq 0.0005$; and obesity: $8 \%$ for non-ICU group vs $12.7 \%$ for ICU group, $p=0.048$ ). Fourteen $(6.8 \%)$ of the 205 cases in the ICU group had a medical comorbidity of sickle cell disease $(p=0.039)$. Compared with the patients in the ICU group, a greater percentage of patients in the non-ICU group presented to the hospital with the COVID-19 infection asymptomatically (18.3 vs $1.9 \%, p \leq 0.0005)$. Also, ICU patients presented with more cough ( 71 vs $41 \%, p \leq 0.0005$ ), fever ( 28 vs $57 \%, p \leq 0.0005$ ), shortness of breath (30 vs $55 \%$, $p=0.000$ ) fatigue ( 5 vs $15 \%, p=0.011$ ), and dizziness ( 0.6 vs $6 \%, p=0.024)$. ICU patients experienced a little less vomiting compared to the non-ICU group (3 vs $1.9 \%$, $p=0.011$ ) and shorter time from exposure to the onset of illness (5.9 vs 4.5 mean days; $p=0.041$ ).

\section{Laboratory and chest $\mathrm{CT}$ findings}

Patients admitted to the ICU in this study were more likely to present with higher levels of the following: white blood cell count $\left(6.9 \times 10^{9} / \mathrm{L}\right.$ vs $\left.10.3 \times 10^{9} / \mathrm{L} ; p \leq 0.0005\right)$, neutrophil count $\left(3.2 \times 10^{9} / \mathrm{L}\right.$ vs $\left.4.9 \times 10^{9} / \mathrm{L} ; p \leq 0.0005\right)$, alanine aminotransferase and aspartate aminotransferase (43 vs $92 \mathrm{U} / \mathrm{L}, p \leq 0.0005$; and 66 vs $109 \mathrm{U} / \mathrm{L}, p \leq 0.0005$; respectively), lactate dehydrogenase level (14 U/L vs $22 \mathrm{U} / \mathrm{L} ; \mathrm{p} \leq 0.0005)$, and ferritin (502 vs $1,647 \mu \mathrm{g} / \mathrm{L}$; $p \leq 0.0005)$. ICU group had lower hemoglobin levels (12.5 vs $11.3 \mathrm{~g} / \mathrm{dL} ; p \leq 0.0005)$; and shown lower rate of normal chest CT images (35 vs $5, p \leq 0.0005$ ), and more major bilateral lung abnormalities (35 vs 69\%; $p \leq 0.0005)$. Other investigations of the cohort are indicated in Table 2.

\section{Pharmacotherapy agents}

As shown in Table 3, the six most prescribed medications were enoxaparin $(56 \% ; n=571)$, paracetamol $(35 \%$; $n=355)$, favipiravir (34\%; $n=353)$, azithromycin (33\%; $n=337)$, ceftriaxone $(32 \% ; n=332)$, and vitamin C (31\%; $n=322$ ). Those patients admitted to the ICU were more likely to have been prescribed the favipiravir ( 54 vs $29 \%$; $p=0.000$ ), azithromycin (54 vs $27 \%$; $\leq 0.0005)$, tocilizumab ( 29 vs $10 \% ; p \leq 0.0005$ ), lopinavir/ritonavir (16 vs $5 \%$; $p \leq 0.0005$ ), dexamethasone (34 vs $16 \% ; \mathrm{p} \leq 0.0005$ ) and methylprednisolone (22 vs $10 \% ; p \leq 0.0005$ ).

\section{Treatment outcomes}

Clinical outcomes of the cohort with mortality documented in $120(11.8 \%)$ of the patients while 778 (76.7\%) of the study cohort recovered. High mortality rate was associated with those that were admitted to an ICU (27.8 vs $7.8 \% ; p \leq 0.0005$ ), if patient had comorbidities (6.3 vs $0.8 \% ; p \leq 0.0005)$, had cerebrovascular disease (16.7 vs $3.5 \% ; p=0.04$ ), had diabetes ( 8.7 vs $1.9 \% ; p \leq 0.0005)$, had hypertension ( 7 vs $2.4 \% ; p=0.003$ ), if patient was symptomatic (5.2 vs $0 \% ; p \leq 0.0005)$, if patient had respiratory rate $>24$ breaths per minute ( 10.8 vs $0 \% ; p \leq 0.0005)$, older age ( $\geq 51$ years) ( 25 vs $2.3 \% ; p \leq 0.0005)$, and those with platelet count $<100 \times 10^{9} / \mathrm{L}$ (37 vs $10.6 \% ; p \leq 0.0005$ ).

\section{Discussion}

We describe the clinical characteristics, laboratory parameters, treatments and outcomes of 1,014 laboratory-confirmed COVID-19 patients admitted to six Ministry of Health's different hospitals in Alahsa Governorate, Saudi Arabia, with a focus on ICU and non-ICU admission.

Based on previously published studies [16, 17], evidence suggests that older male patients are the most susceptible to infection of COVID-19, which was supported by our data. This might be attributed mainly to the differences in the inclusion criteria and the population groups of age in the current study or can be explained by the gender-based biological differences 
Table 1 Demographic and clinical characteristics of 1,014 patients with COVID-19 hospitalized in Alahsa Governorate, Saudi Arabia, stratified by ICU admission

\begin{tabular}{|c|c|c|c|c|}
\hline Characteristic & All $(n=1014)$ & Non-ICU $(n=809)$ & ICU $(n=205)$ & $p$-value \\
\hline \multicolumn{5}{|l|}{ Demographics } \\
\hline Age, mean $\pm S D$, years & $47.2 \pm 19.3(1-104)$ & $45.3 \pm 19.3(1-104)$ & $52.9 \pm 17.3(5-95)$ & $\leq 0.0005$ \\
\hline \multicolumn{5}{|l|}{ Distribution } \\
\hline $0-10$ years & $28(2.8 \%)$ & $20(2.5 \%)$ & $8(3.9 \%)$ & \multirow[t]{10}{*}{$\leq 0.0005$} \\
\hline $11-20$ years & $37(3.6 \%)$ & $33(4.2 \%)$ & $4(1.9 \%)$ & \\
\hline $21-30$ years & $126(12.5 \%)$ & $110(13.6 \%)$ & $16(7.8 \%)$ & \\
\hline $31-40$ years & $214(21.1 \%)$ & $195(24.1 \%)$ & $19(9.3 \%)$ & \\
\hline $41-50$ years & $164(16.2 \%)$ & $137(16.9 \%)$ & $27(13.2 \%)$ & \\
\hline $51-60$ years & $186(18.3 \%)$ & $155(19.2 \%)$ & $31(15.1 \%)$ & \\
\hline $61-70$ years & $144(14.2 \%)$ & $108(13.3 \%)$ & $36(17.6 \%)$ & \\
\hline $71-80$ years & $65(6.4 \%)$ & $32(3.9 \%)$ & $33(16.1 \%)$ & \\
\hline $81-90$ years & $37(3.6 \%)$ & $10(1.2 \%)$ & $27(13.2 \%)$ & \\
\hline$\geq 90$ years & $13(1.3 \%)$ & $9(1.1 \%)$ & $4(1.9 \%)$ & \\
\hline \multicolumn{5}{|l|}{ Gender } \\
\hline Male & $582(57.4 \%)$ & $466(57.6 \%)$ & $116(56.5 \%)$ & \multirow[t]{2}{*}{0.858} \\
\hline Female gender & $432(42.6 \%)$ & $343(42.4 \%)$ & $89(43.5 \%)$ & \\
\hline \multicolumn{5}{|l|}{ Nationality } \\
\hline Saudi & 900 (88.8\%) & $721(89.1 \%)$ & $179(87.3 \%)$ & \multirow[t]{2}{*}{0.886} \\
\hline Non-Saudi & $114(11.2 \%)$ & $88(10.9 \%)$ & $26(12.7 \%)$ & \\
\hline \multicolumn{5}{|l|}{ Occupation } \\
\hline Healthcare worker & $21(2.1 \%)$ & $16(2 \%)$ & $5(2.4 \%)$ & \multirow[t]{2}{*}{0.755} \\
\hline Non-healthcare worker & $993(97.9 \%)$ & 793 (98\%) & $200(97.6 \%)$ & \\
\hline \multicolumn{5}{|l|}{ Smoking status } \\
\hline Not a smoker & $920(90.7 \%)$ & $786(97.2 \%)$ & $134(65.4 \%)$ & \multirow[t]{3}{*}{0.223} \\
\hline Past smoker & $35(3.4 \%)$ & $6(0.7 \%)$ & $29(14.1 \%)$ & \\
\hline Current smoker & $59(5.9 \%)$ & $17(2.1 \%)$ & $42(20.5 \%)$ & \\
\hline \multicolumn{5}{|l|}{ Hospital } \\
\hline King Fahad Hofuf Hospital & $300(29.6 \%)$ & $249(30.8 \%)$ & $51(24.9 \%)$ & \multirow[t]{6}{*}{$\leq 0.0005$} \\
\hline Aljabr Eye and ENT Hospital & $218(21.5 \%)$ & $174(21.5 \%)$ & $44(21.5 \%)$ & \\
\hline Alomran General Hospital & $200(19.7 \%)$ & $150(18.5 \%)$ & $50(24.4 \%)$ & \\
\hline King Faisal General Hospital & $163(16.1 \%)$ & $152(18.8 \%)$ & $11(5.4 \%)$ & \\
\hline Maternity and Children Hospital & $71(7 \%)$ & $55(6.8 \%)$ & $16(7.8 \%)$ & \\
\hline Prince Saud Bin Jalawi Hospital & $62(6.1 \%)$ & $29(3.6 \%)$ & $33(16 \%)$ & \\
\hline \multicolumn{5}{|l|}{ Educational level } \\
\hline Illiterate & $146(14.4 \%)$ & $61(7.5 \%)$ & 85 (41.5\%) & \multirow[t]{4}{*}{0.06} \\
\hline Primary to secondary & $529(52.2 \%)$ & $449(55.5 \%)$ & 80 (39\%) & \\
\hline University & $172(17 \%)$ & $153(18.9 \%)$ & $19(9.3 \%)$ & \\
\hline Unknown & $167(16.4 \%)$ & $146(18.1 \%)$ & $21(10.2 \%)$ & \\
\hline \multicolumn{5}{|l|}{ Exposure history } \\
\hline Contact with positive confirmed case & $668(65.9 \%)$ & $548(67.7 \%)$ & $120(58.5 \%)$ & \multirow[t]{5}{*}{0.062} \\
\hline Familiar/cluster infections & $180(17.8 \%)$ & $149(18.4 \%)$ & $31(15.1 \%)$ & \\
\hline Hospital staff & $21(2.1 \%)$ & $17(2.1 \%)$ & $4(1.9 \%)$ & \\
\hline From outside Saudi & $1(0.1 \%)$ & $1(0.1 \%)$ & 0 & \\
\hline Unknown & $144(14.1 \%)$ & $94(11.6 \%)$ & $50(24.4 \%)$ & \\
\hline \multicolumn{5}{|l|}{ Highest temperature $\left({ }^{\circ} \mathrm{C}\right)$ : } \\
\hline$<37.3$ & $467(46.1 \%)$ & $414(51.3 \%)$ & $53(25.8 \%)$ & \multirow[t]{3}{*}{$\leq 0.0005$} \\
\hline $37.3-38.0$ & $338(33.3 \%)$ & $226(27.9 \%)$ & $112(54.7 \%)$ & \\
\hline $38.01-39.0$ & $147(14.5 \%)$ & $116(14.3 \%)$ & $31(15.1 \%)$ & \\
\hline$>39.0$ & $62(6.1 \%)$ & $53(6.5 \%)$ & $9(4.4 \%)$ & \\
\hline
\end{tabular}


Table 1 (continued)

\begin{tabular}{|c|c|c|c|c|}
\hline Characteristic & All $(n=1014)$ & Non-ICU $(n=809)$ & $\operatorname{ICU}(n=205)$ & $p$-value \\
\hline Respiratory rate $>24$ breaths per min & $312(30.8 \%)$ & $168(20.8 \%)$ & $144(70.2 \%)$ & $\leq 0.0005$ \\
\hline \multicolumn{5}{|l|}{ Comorbidities } \\
\hline Diabetes & $202(19.9 \%)$ & $142(17.5 \%)$ & $60(29.3 \%)$ & $\leq 0.0005$ \\
\hline Hypertension & $276(27.2 \%)$ & 165 (20.4\%) & $111(54.1 \%)$ & $\leq 0.0005$ \\
\hline Obesity $\left(\mathrm{BMI} \geq 30 \mathrm{~kg} / \mathrm{m}^{2}\right)$ & $91(9 \%)$ & $65(8 \%)$ & $26(12.7 \%)$ & 0.048 \\
\hline Asthma & $26(2.6 \%)$ & $15(1.8 \%)$ & $11(5.4 \%)$ & 0.179 \\
\hline Coronary artery disease & $16(1.6 \%)$ & $7(0.9 \%)$ & $9(4.4 \%)$ & 0.778 \\
\hline Thalassemia & $3(0.3 \%)$ & 0 & $3(1.5 \%)$ & 0.222 \\
\hline Chronic kidney disease & $5(0.5 \%)$ & 0 & $5(2.4 \%)$ & 0.592 \\
\hline Liver disease & $1(0.1 \%)$ & 0 & $1(0.5 \%)$ & 0.778 \\
\hline Cerebrovascular disease & $6(0.6 \%)$ & $3(0.4 \%)$ & $3(1.5 \%)$ & 0.127 \\
\hline Cancer & $5(0.5 \%)$ & $1(0.1 \%)$ & $4(1.9 \%)$ & 0.691 \\
\hline Cardiomyopathies & $3(0.3 \%)$ & $1(0.1 \%)$ & $2(1 \%)$ & 0.395 \\
\hline Heart failure & $2(0.2 \%)$ & 0 & $2(1 \%)$ & 0.605 \\
\hline Ischemic heart disease & $12(1.2 \%)$ & $3(0.4 \%)$ & $9(4.4 \%)$ & 0.520 \\
\hline Sickle cell disease & $31(3 \%)$ & $17(2.1)$ & $14(6.8 \%)$ & 0.039 \\
\hline G6PD deficiency & $14(1.4 \%)$ & $11(1.3 \%)$ & $3(1.5 \%)$ & 0.621 \\
\hline Pregnant & $18(1.8 \%)$ & $10(1.2 \%)$ & $8(3.9 \%)$ & 0.379 \\
\hline Asymptomatic & $152(15 \%)$ & $148(18.3 \%)$ & $4(1.9 \%)$ & $\leq 0.0005$ \\
\hline \multicolumn{5}{|l|}{ Symptoms } \\
\hline Fever & $348(34.3 \%)$ & $230(28.4 \%)$ & $118(57.6 \%)$ & $\leq 0.0005$ \\
\hline Shortness of breath & $362(35.7 \%)$ & $248(30.6 \%)$ & $114(55.6 \%)$ & $\leq 0.0005$ \\
\hline Cough & $484(47.7 \%)$ & $338(41.8 \%)$ & $146(71.2 \%)$ & $\leq 0.0005$ \\
\hline Aches and pains & $43(4.2 \%)$ & $24(3 \%)$ & $19(9.3 \%)$ & 0.139 \\
\hline Loss of taste or smell & $54(5.3 \%)$ & $38(4.7 \%)$ & $16(7.8 \%)$ & 0.562 \\
\hline Nausea & 19 (1.9\%) & $14(1.7 \%)$ & $5(2.4 \%)$ & 0.170 \\
\hline Chest pain & $42(4.1 \%)$ & $26(3.2 \%)$ & $16(7.8 \%)$ & 0.258 \\
\hline Sore throat & $46(4.5 \%)$ & 27 (3.3\%) & $19(9.3 \%)$ & 0.053 \\
\hline Headache & $70(6.9 \%)$ & $46(5.7 \%)$ & $24(11.7 \%)$ & 0.063 \\
\hline Diarrhea & $70(6.9 \%)$ & $62(7.7 \%)$ & $8(3.9 \%)$ & 0.077 \\
\hline Fatigue & $74(7.3 \%)$ & $42(5.2 \%)$ & $32(15.6 \%)$ & 0.011 \\
\hline Myalgia & $68(6.7 \%)$ & $44(5.4 \%)$ & $24(11.7 \%)$ & 0.153 \\
\hline Abdominal pain & $25(2.5 \%)$ & $19(2.3 \%)$ & $6(2.9 \%)$ & 0.102 \\
\hline Vomiting & $28(2.8 \%)$ & $24(3 \%)$ & $4(1.9 \%)$ & 0.013 \\
\hline Anorexia & $39(3.8 \%)$ & $27(3.3 \%)$ & $12(5.8 \%)$ & 0.399 \\
\hline Rash & $12(1.1 \%)$ & $9(1.1 \%)$ & $3(1.5 \%)$ & 0.470 \\
\hline Dizziness & $18(1.8 \%)$ & $5(0.6 \%)$ & $13(6.3 \%)$ & 0.024 \\
\hline Time from exposure to the onset of illness (days) & $5.39 \pm 3.8(1-15)$ & $5.98 \pm 3.96(1-14)$ & $4.49 \pm 3.5(2-15)$ & 0.041 \\
\hline Time from illness onset to first hospital admission (days) & $5.99 \pm 3.9(1-30)$ & $5.97 \pm 3.7(1-22)$ & $6.04 \pm 4.45(1-30)$ & 0.646 \\
\hline
\end{tabular}

Data are presented as number (\%), or mean \pm SD and (minimum-maximum)

COVID-19 coronavirus disease 2019, ICU intensive care unit, G6PD Glucose-6-phosphate dehydrogenase, BMI body mass index, SD standard deviation

in the host immune response to COVID-19 infection. Additionally, gender-based biological factors underlying the immune response are important determinants of susceptibility to SARS-CoV-2 infection, disease, and mortality outcomes $[21,22]$. In our study, males gender predominated admission to ICU, a finding suggested in previous reports [23-25] and in contradiction with data for other cohorts suggesting an equal proportion of ICU admission for both genders [11, 12]. However, this was probably related to different lifestyles between men and women, stronger immune response to infections in females who outlive men [26] or lower rates 


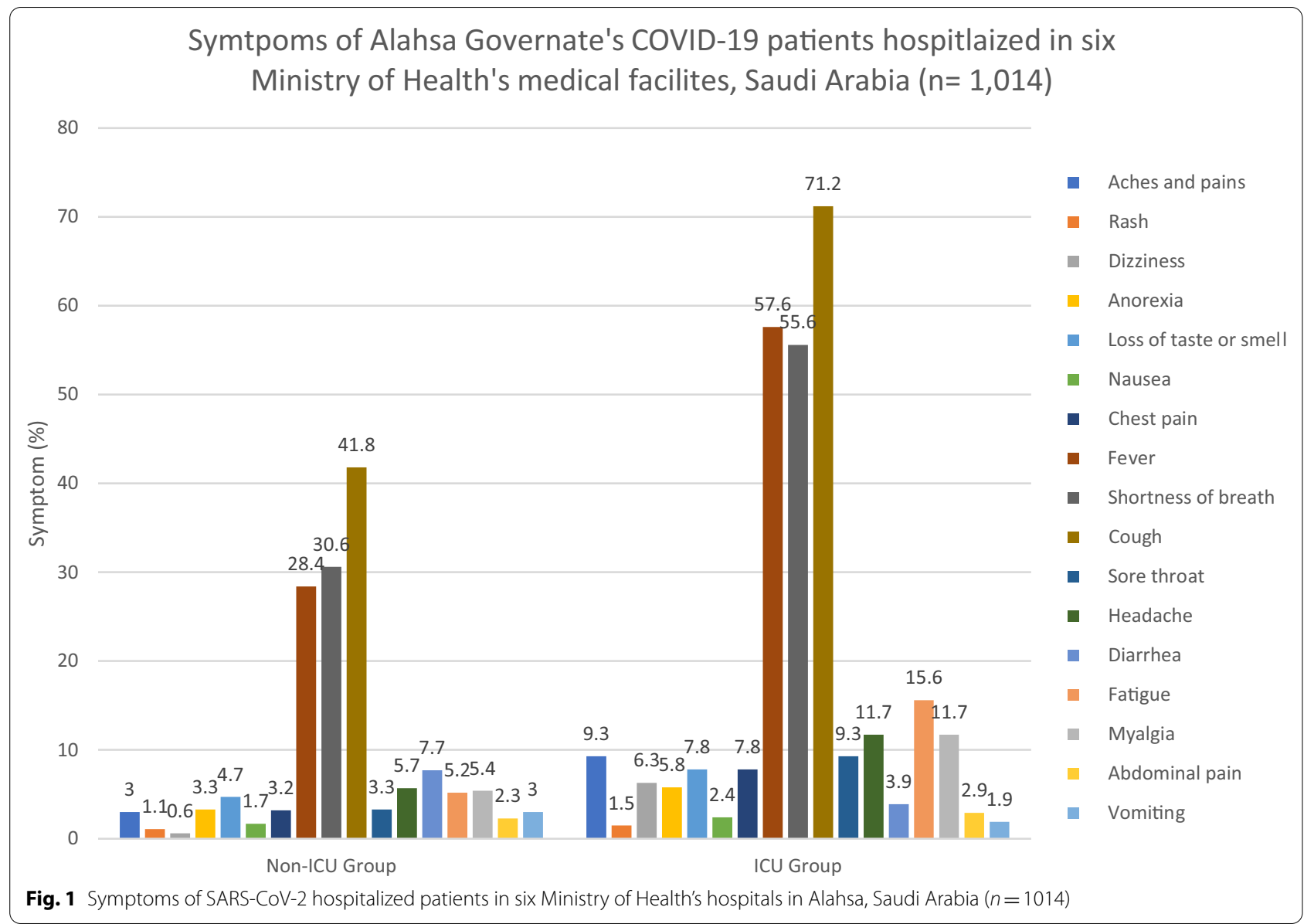

of healthcare service utilization by males [27]. Moreover, some authors explained this gender difference with higher rates of comorbidities among men [28], prior studies suggested a higher trend among females to follow hand hygiene [29] and preventive care [14, 30]. The mean age of ICU patients was $52 \pm 17$ years; similar to one small cohort in Oman State [31] and a larger cohort in Kuwait [32]; and lower than reports from large cohorts in Italy [25] and China [24]. Age appears to be the major risk factor that predicts progression of COVID-19 patients to acute respiratory distress syndrome (ARDS) and need for ICU admission $[33,34]$; however, there were no statistically significant differences in age between ICU and non-ICU groups in our study. Importantly, adults of any age may develop severe disease and experience adverse outcomes, especially those with comorbidities [35].

In our cohort, most of the patients were not smokers. Nevertheless, cases who were admitted to the ICU in this study represent more than $70 \%$ of all the patients who were smoking currently. Smoking has been indicated as a risk factor in some previously published reports and predict progression and worsening from COVID-19 [24, 36].
In both non-ICU and ICU groups, a high percentage of all patients showed abnormal bilateral chest CT images consistent with pneumonia and/or ARDS. Chest CT abnormalities in COVID-19 patients as shown in other studies are often bilateral, have a peripheral distribution, and involve the lungs lower lobes [37, 38]. Though some chest CT findings may be characteristic of COVID-19, no finding can completely rule out or rule in the possibility of COVID-19. Use of chest CT for screening or diagnosis of SARS-CoV-2 is not recommended and chest radiographs should be reserved when there is an indication (e.g., catheter- or endotracheal tube-placement or a relevant clinical change is recommended [39]; this rationale is based upon the increased risk of viral shedding with procedures that require transfer out of the ICU. It is worth mentioning that among ICU patients who clinically improve, resolution of radiographic abnormalities may delay improvements in fever and hypoxia [40].

Fever, shortness of breath and cough were the most common symptoms in all patients and strong predictors for the patient's admission to ICU. Similar findings have been reported in previous studies [16, 17]; however, fever and cough are not reliable indicators to confirm 
Table 2 Laboratory and chest radiography findings in 1,014 patients with COVID-19 admitted to six hospitals in Alahsa Governorate, Saudi Arabia, stratified by ICU admission

\begin{tabular}{|c|c|c|c|c|}
\hline Characteristic & All $(n=1014)$ & Non-ICU $(n=809)$ & ICU $(n=205)$ & $p$-value \\
\hline \multicolumn{5}{|l|}{ Laboratory findings } \\
\hline White blood cell count $\left(\times 10^{9} / L\right)^{a}$ & $7.7 \pm 4.5(1.1-42.9)$ & $6.9 \pm 3.3(1.1-26.6)$ & $10.3 \pm 6.7(1.4-42.9)$ & $\leq 0.0005$ \\
\hline \multicolumn{5}{|c|}{ White blood cell count $\left(\times 10^{9} / \mathrm{L}\right)(\mathrm{No}(\%))^{\mathrm{a}}$} \\
\hline$<4$ & $150(14.8 \%)$ & $127(15.7 \%)$ & $23(11.3 \%)$ & \multirow[t]{3}{*}{$\leq 0.0005$} \\
\hline $4-10$ & $651(64.2 \%)$ & $555(68.6 \%)$ & $96(46.8 \%)$ & \\
\hline$>10$ & $213(21 \%)$ & $127(15.7 \%)$ & $86(41.9 \%)$ & \\
\hline Neutrophil count $\left(\times 10^{9} / L\right)^{b}$ & $3.4 \pm 3.2(0.01-9.7)$ & $3.2 \pm 3.3(0.01-9.6)$ & $4.9 \pm 3.6(0.1-9.7)$ & $\leq 0.0005$ \\
\hline Lymphocyte count $\left(\times 10^{9} /\llcorner)^{b}\right.$ & $1.3 \pm 1.6(0.3-8.4)$ & $1.2 \pm 1.6(0.34-8.4)$ & $1.3 \pm 1.5(0.3-6.2)$ & 0.278 \\
\hline \multicolumn{5}{|l|}{ Lymphocyte count $\left(\times 10^{9} / \mathrm{L}\right)(\mathrm{No}(\%))^{\mathrm{a}}$} \\
\hline$<1.0$ & $195(19.2 \%)$ & $131(16.2 \%)$ & $64(31.2 \%)$ & \multirow[t]{2}{*}{0.138} \\
\hline$\geq 1.0$ & $819(80.8 \%)$ & $678(83.8 \%)$ & $141(68.8 \%)$ & \\
\hline Hemoglobin $(\mathrm{g} / \mathrm{dL})^{\mathrm{b}}$ & $12.2 \pm 2.3(3.7-17.4)$ & $12.5 \pm 2.2(4.5-17.4)$ & $11.3 \pm 2.3(3.7-16.4)$ & $\leq 0.0005$ \\
\hline Platelet count $\left(\times 10^{9} / L\right)^{b}$ & $285.3 \pm 130.6(8-861)$ & $285.6 \pm 123.1(8-802)$ & $290.2 \pm 153.3(24-861)$ & 0.850 \\
\hline \multicolumn{5}{|l|}{ Platelet count $\left(\times 10^{9} / \mathrm{L}\right)(\mathrm{No}(\%))^{\mathrm{a}}$} \\
\hline$<100$ & $80(7.9 \%)$ & $57(7.1 \%)$ & $23(11.2 \%)$ & \multirow[t]{2}{*}{0.128} \\
\hline$\geq 100$ & $934(92.1 \%)$ & $752(92.9 \%)$ & $182(88.8 \%)$ & \\
\hline Alanine aminotransferase $(U / L)^{b}$ & $55.9 \pm 144.3(3.8-2,465)$ & $43.2 \pm 84(3.8-1,534)$ & $92.6 \pm 227.6(5.7-2,465)$ & $\leq 0.0005$ \\
\hline Aspartate aminotransferase $(\mathrm{U} / \mathrm{L})^{\mathrm{b}}$ & $80.1 \pm 432.8(3-10,252)$ & $66.8 \pm 463.5(3-10,252)$ & $109.5 \pm 283(7.3-2,870)$ & $\leq 0.0005$ \\
\hline \multicolumn{5}{|c|}{ Aspartate aminotransferase (U/L) (No (\%)) ${ }^{\mathrm{a}}$} \\
\hline$<40$ & $704(69.4 \%)$ & $592(73.2 \%)$ & $112(54.6 \%)$ & \multirow[t]{2}{*}{$\leq 0.0005$} \\
\hline$\geq 40$ & $310(30.6 \%)$ & $217(26.8 \%)$ & $93(45.4 \%)$ & \\
\hline Potassium $(\mathrm{mmol} / \mathrm{L})^{\mathrm{b}}$ & $4.4 \pm 0.7(2.8-8.1)$ & $4.4 \pm 0.7(2.8-8.1)$ & $4.5 \pm 0.7(2.9-7.5)$ & 0.132 \\
\hline Sodium (mmol/L) & $137.9 \pm 5.7(57.8-163)$ & $137.7 \pm 5.4(57.8-163)$ & $138.8 \pm 6.5(127-163)$ & 0.960 \\
\hline Creatinine $(\mu \mathrm{mol} / \mathrm{L})^{\mathrm{b}}$ & $89 \pm 96.5(5.2-927)$ & $84.5 \pm 92.7(5.7-927)$ & $92.5 \pm 81.4(5.2-645)$ & 0.081 \\
\hline \multicolumn{5}{|l|}{ Creatinine $(\mu \mathrm{mol} / \mathrm{L})\left(\mathrm{No}(\%)^{\mathrm{a}}\right.$} \\
\hline$\leq 107$ & $833(82.1 \%)$ & $673(83.2 \%)$ & $160(78.1 \%)$ & \multirow[t]{2}{*}{0.002} \\
\hline$>107$ & $181(17.9 \%)$ & $136(16.8 \%)$ & $45(21.9 \%)$ & \\
\hline Creatinine kinase $(\mathrm{U} / \mathrm{L})^{\mathrm{b}}$ & $257.6 \pm 821(2.2-9,716)$ & $204 \pm 673.4(2.2-9,716)$ & $497.4 \pm 1,314(9-9,716)$ & 0.098 \\
\hline \multicolumn{5}{|l|}{ Creatinine kinase (U/L) (No (\%)) ${ }^{a}$} \\
\hline$\leq 198$ & $680(67.1 \%)$ & $557(68.8 \%)$ & $123(60.2 \%)$ & \multirow[t]{2}{*}{0.015} \\
\hline$>198$ & $334(32.9 \%)$ & $252(31.2 \%)$ & $82(39.8 \%)$ & \\
\hline Lactate dehydrogenase $(\mathrm{U} / \mathrm{L})^{c}$ & $16.4 \pm 20.1(0.2-96)$ & $14.2 \pm 18.7(0.2-96)$ & $22.6 \pm 24.2(2.1-96)$ & $\leq 0.0005$ \\
\hline \multicolumn{5}{|l|}{ Lactate dehydrogenase (U/L) (No (\%)) } \\
\hline$\leq 280$ & $549(54.1 \%)$ & $479(59.2 \%)$ & $70(34.2 \%)$ & \multirow[t]{2}{*}{$\leq 0.0005$} \\
\hline$>280$ & $465(45.9 \%)$ & $330(40.8 \%)$ & $135(65.8 \%)$ & \\
\hline C-reactive protein $(\mathrm{mg} / \mathrm{L})^{\mathrm{b}}$ & $16.4 \pm 20.1(0.2-96)$ & $14.2 \pm 18.7(0.2-96)$ & $22.6 \pm 24.2(2.1-96)$ & 0.092 \\
\hline Ferritin $(\mu \mathrm{g} / \mathrm{L})^{c}$ & $845.6 \pm 3,430(2-49,813)$ & $502.9 \pm 860.5(4.4-7,945)$ & $1,647 \pm 5,270(2-42,969)$ & $\leq 0.0005$ \\
\hline ESR $(m m / \text { hour })^{b}$ & $49 \pm 35.8(1-139)$ & $47.9 \pm 35.2(1-139)$ & $54.3 \pm 38.7(1-130)$ & 0.552 \\
\hline \multicolumn{5}{|l|}{ Chest $C T$ images $^{a}$} \\
\hline Normal & $295(29.1 \%)$ & $283(35 \%)$ & $12(5.8 \%)$ & \multirow[t]{5}{*}{$\leq 0.0005$} \\
\hline Abnormal bilateral lung & $427(42.1 \%)$ & $285(35.2 \%)$ & $142(69.3 \%)$ & \\
\hline Abnormal right lung & $95(9.4 \%)$ & $79(9.8 \%)$ & $16(7.8 \%)$ & \\
\hline Abnormal left lung & $110(10.9 \%)$ & $96(11.9 \%)$ & $14(6.8 \%)$ & \\
\hline No radiography data available & $87(8.5 \%)$ & $66(8.1 \%)$ & $21(10.2 \%)$ & \\
\hline
\end{tabular}

Data are presented as number (\%), or mean $\pm \mathrm{SD}$ and (minimum-maximum)

COVID-19 coronavirus disease 2019, ESR erythrocyte sedimentation rate, ICU intensive care unit, SD standard deviation

${ }^{\text {a }}$ Chi-square $\left(X^{2}\right)$ test was used to compare between non-ICU and ICU groups

${ }^{b}$ Mann-Whitney $U$ test was used to compare between non-ICU and ICU groups

'Student's $t$-test was used to compare between non-ICU and ICU groups

* Represents significant differences 
Table 3 Treatments and outcomes in 1,014 patients with COVID-19 admitted to six hospitals in Alahsa Governorate, Saudi Arabia, stratified by ICU admission

\begin{tabular}{|c|c|c|c|c|}
\hline Characteristic & All $(n=1014)$ & Non-ICU $(n=809)$ & ICU $(n=205)$ & $p$-value \\
\hline No pharmacotherapy was given & $115(11.3 \%)$ & 88 (10.9\%) & $27(13.2 \%)$ & 0.338 \\
\hline \multicolumn{5}{|l|}{ Pharmacotherapy used } \\
\hline Hydroxychloroquine & $269(26.5 \%)$ & 235 (29\%) & $34(16.6 \%)$ & $\leq 0.0005$ \\
\hline Azithromycin & $337(33.2 \%)$ & $226(27.9 \%)$ & $111(54 \%)$ & $\leq 0.0005$ \\
\hline Oseltamivir & $88(8.7 \%)$ & $73(9 \%)$ & $15(7.3 \%)$ & 0.385 \\
\hline Vitamin C & $322(31.7 \%)$ & $241(29.8 \%)$ & 81 (39.5\%) & 0.029 \\
\hline Vitamin D & $286(28.2 \%)$ & $211(26.1 \%)$ & $75(36.6 \%)$ & 0.001 \\
\hline Zinc & $195(19.2 \%)$ & $142(17.5 \%)$ & $53(25.8 \%)$ & 0.001 \\
\hline Ceftriaxone & $332(32.7 \%)$ & $257(31.8 \%)$ & 75 (36.6\%) & 0.08 \\
\hline Enoxaparin & $571(56.3 \%)$ & $473(58.5 \%)$ & $98(47.8 \%)$ & 0.005 \\
\hline Favipiravir & $353(34.8 \%)$ & $241(29.8 \%)$ & $112(54.6 \%)$ & $\leq 0.0005$ \\
\hline Thiamine & $94(9.3 \%)$ & $78(9.6 \%)$ & $16(7.8 \%)$ & 0.467 \\
\hline Interferon beta & $53(5.2 \%)$ & $33(4.1 \%)$ & $20(9.7 \%)$ & 0.002 \\
\hline Lopinavir/ritonavir & $80(7.9 \%)$ & $47(5.8 \%)$ & $33(16.1 \%)$ & $\leq 0.0005$ \\
\hline Dexamethasone & $202(19.9 \%)$ & $131(16.2 \%)$ & $71(34.8 \%)$ & $\leq 0.0005$ \\
\hline Hydrocortisone & $84(8.3 \%)$ & $58(7.2 \%)$ & $26(12.7 \%)$ & $\leq 0.0005$ \\
\hline Methylprednisolone & $135(13.3 \%)$ & $88(10.9 \%)$ & 47 (22.9\%) & $\leq 0.0005$ \\
\hline Tocilizumab & $144(14.2 \%)$ & $83(10.2 \%)$ & $61(29.7 \%)$ & $\leq 0.0005$ \\
\hline Paracetamol & $355(35 \%)$ & $268(33.1 \%)$ & 87 (42.4\%) & $\leq 0.0005$ \\
\hline \multicolumn{5}{|l|}{ Prognosis } \\
\hline Hospitalization & $87(8.6 \%)$ & $62(7.7 \%)$ & 25 (12.2\%) & $\leq 0.0005$ \\
\hline Transferred & $29(2.9 \%)$ & $17(2.1 \%)$ & $12(5.8 \%)$ & \\
\hline Recovered & $778(76.7 \%)$ & $667(82.4 \%)$ & $111(54.1 \%)$ & \\
\hline Died & $120(11.8 \%)$ & $63(7.8 \%)$ & $57(27.8 \%)$ & \\
\hline
\end{tabular}

Data are presented as number (\%)

COVID-19 coronavirus disease 2019 , ICU intensive care unit

the diagnosis of COVID-19 or rule out other differential diagnoses [41], and shortness of breath usually emerges a few days after these initial symptoms. Therefore, it is difficult based on fever, cough and shortness of breath symptoms alone to distinguish COVID-19 from other bacterial or viral infections [42]. While high fever was associated with a higher likelihood of developing ARDS and admission to ICU [35], it appears to be associated with a lower mortality rate [33, 43].

In line with our results, respiratory rate has also been identified as an important predictor of ICU admission in patients with COVID-19 $[9,44,45]$. Frequent monitoring and documentation of the respiratory rate, along with education on appropriate action when the respiratory rate is fluctuating, may help to identify and manage COVID-19 patients at risk and accordingly reduce the incidence of serious adverse events.

Consistent with other studies, very common comorbidities to predict severity of COVID-19 and ICU admission were diabetes $[10,24,25,35,46,47]$, hypertension $[10,24,25,47]$, obesity $[10,48,49]$, cardiovascular disease [24, 35, 43], chronic kidney disease [24, 25, 43], cerebrovascular disease [43, 50,51], and sickle cell disease [52-54]. COVID-19 patients with these underlying comorbidities were more likely to develop into critically severe cases, require respiratory support treatment, and eventually be transferred to ICU.

The ICU admission rate for COVID-19 patients in our study was $20 \%$ and identical to the rates reported in a Kuwaiti study [32] and a report on the analysis of a second surge in Houston, Texas, USA [10]. Higher ICU admission rates in COVID-19 cohorts were reported; for instance, in Chinese cohorts, rates of ICU admission ranged from 7 to $26 \%[9,14,15,43]$, and in a large US cohort (a total of 4,226 COVID-19 cases) reported to CDC from a total of 49 states, the District of Columbia, and three U.S. territories during February 12-March 16, 2020, ICU admission rate was 53\% [55]; and there was a lower proportion of ICU admission of all hospitalized COVID-19 patients based on studies in Italy (16\%) [12, 13]. Rates of ICU admission differ due to different healthcare systems, medical practice and admission criteria as 
well as differences in predisposing factors such as age, comorbidities and testing availability in the patients served.

We report similar results of previous studies regarding the laboratory abnormalities that have been described in severe COVID-19 patients who necessitated ICU admission including elevated white blood cells $[9,32,56]$, neutrophils [9, 32, 56, 57], alanine aminotransferase and aspartate aminotransferase $[9,24,32,35,45,56,58]$, creatinine $[9,32,35,43,45]$, creatinine kinase $[9,35,45,56$, $58]$, lactate dehydrogenase $[9,24,31,32,35,43,45,56$, $58,59]$, and ferritin $[24,31,45,56,58,59]$. Continuous tracking of laboratory findings during the management of COVID-19 cases is crucial to early identify those patients who may progress to severe status.

We report taste and smell disturbances as a rare clinical finding in our cohort on COVID-19; however, several authors reported different findings [60-62]. A metaanalysis demonstrated a prevalence of $52.73 \%$ (95\% CI: 29.64-75.23\%) and 43.93\% (95\% CI: 20.46-68.95\%) for smell and taste dysfunction among COVID-19 patients, reaching $86.60 \%$ in studies that used validated instruments [63]. In particular, these symptoms seem to be common in the early stages of the disease and in paucisymptomatic patients [64]. It was hypothesized that COVID-19 patients under-report the frequency of taste and smell disorders, and authors suggested that those with more severe SARS-CoV-2 disease neglect such symptoms in the setting of severe respiratory disease [60, 61].

Optimal approaches to treat patients hospitalized with COVID-19 are lacking. Strategies to manage patients infected with COVID-19 are based on limited data and changes rapidly as clinical data emerge. Lack of welldefined management plan for COVID-19 disease results in the use of various treatment and adjuvant therapies during hospital stay. Many antiretroviral, anti-malarial and anti-inflammatory medications were tried on hundreds of thousands of affected patients to find effective therapeutics and to reduce the spread of this global pandemic. For mild to moderate COVID-19 cases, care is mostly supportive, with close monitoring for disease progression. Supportive measures were given to all patients in the present study which included but not limited to respiratory support, circulatory support, prevention of secondary infections, and preservation of renal, hepatic, and neurological function. In addition to the implementation of the basic principles of critical care medicine, patients in the current cohort also received pharmacologic prophylaxis for venous thromboembolism. For patients who are receiving supplemental oxygen (including those who are on high-flow oxygen and noninvasive ventilation), low-dose dexamethasone and, if available, remdesivir is/are suggested [65, 66]. However, the optimal role of remdesivir remains uncertain, and some guidelines panels recommend not using it in hospitalized patients; including the WHO; as there is no clear evidence that it improves patient-important outcomes of COVID-19 hospitalized patients such as mortality and need for mechanical ventilation. Lopinavir-ritonavir is a human immunodeficiency virus antiviral combination that has been considered for the treatment of human viral corona respiratory infections, including Middle East Respiratory Syndrome (MERS-CoV) and COVID-19 disease $[67,68]$. However, use of lopinavir/ritonavir for treatment of SARS-CoV-2 in hospitalized patients is not recommended as several studies have failed to prove its efficacy [69-71]. Recently, a variety of therapeutic drug candidates with various forms of modes of action have been considered to combat extreme hyperinflammatory responses in hospitalized COVID-19 patients [72]. From a tsunami of upcoming clinical research studies, authors concluded that cytokine antagonists such as tocilizumab and sarilumab and Janus kinase-signal transducer and activator of transcription inhibitors such as ruxolitinib and baricitinib, represent potential candidates in managing severe inflammatory response or cytokine storm [72]. Convalescent plasma therapy, antibody-based therapies, mesenchymal stem cell therapies, and medicinal herbs have shown potential to combat the disease severity, lessen cytokine storm, and reduce mortality in severely ill patients with COVID-19 [72]. It is worth mentioning that initiating therapy earlier is known to be more effective [73], since systemic hyperinflammation rather than viral pathogenicity dominates later stages of SARS-CoV-2 infection. Until sufficient evidence is available, the WHO has warned against physicians and medical associations recommending or administering unproven treatments to patients with SARS-CoV-2 or people self-medicating with them.

\section{Limitation of the study}

Cautions must be taken when generalizing of the current study findings as it has several limitations. First, the retrospective nature of the study design could have introduced some bias due to reliance on clinical medical records. Second, the follow-up was limited through July 30th, 2020, hindering the possibility of including all patients' outcomes as some of them are still remained hospitalized. Therefore, there may have been some bias regarding the patients' prognosis. Third, we were not able to provide details on the radiological characters of our study cohort. Finally, some of the follow-up data were missing in the medical records. Additionally, clinical follow-up data for patients after recovery from COVID19 infection were not available which can be used to 
examine psychological and longer-term functional abnormalities.

\section{Conclusion}

SARS-CoV-2 is a worldwide pandemic and has placed significant surges in demand for acute and critical care services on hospitals in many countries. Old age; body temperatures in the range of $37.3-38.0{ }^{\circ} \mathrm{C}$; respiratory rate of $>24$ bpm, diabetes; hypertension; obesity; sickle cell disease; showing symptoms of COVID-19; fever, shortness of breath, cough, fatigue, vomiting, and dizziness; elevated white blood cells, neutrophils, alanine aminotransferase and alkaline aminotransferase, lactate dehydrogenase, and ferritin; and abnormal bilateral chest CT images were associated with ICU admission. Besides, the prevalence of mortality had a strong relation with ICU admission, comorbidities; if patient had diabetes, hypertension, and cerebrovascular disease; showing symptoms of COVID-19, respiratory rate $>24 \mathrm{bpm}$, old age, and platelet count less than $<100 \times 10^{9} / \mathrm{L}$. Identifying key clinical characteristics of COVID-19 that predict ICU admission and high mortality rate can be useful for frontline healthcare providers in making the right clinical decision under time-sensitive and resource-constricted environment.

\begin{abstract}
Abbreviations
COVID-19: Coronavirus disease 2019; SARS-CoV-2: Severe acute respiratory syndrome coronavirus 2; RT-PCR: Real-time reverse transcription-polymerase chain reaction; CRP: C-reactive protein; ICU: Intensive care unit; G6PD: Glucose6-phosphate dehydrogenase; BMI: Body mass index.
\end{abstract}

\section{Acknowledgements}

The authors would like to acknowledge the Ministry of Health and the Chief Executive Officer for Alahsa Health Cluster for supporting this research. We also would like to extend our thanks to King Fahad Hofuf Hospital, Prince Saud Bin Jalawi Hospital, Maternity and Children Hospital, King Faisal General Hospital, Alomran General Hospital and Aljaber Eye and ENT Hospital for participating in this study and the IRB of KFHH for ethical approval of the study.

\section{Authors' contributions}

SA, AA, ZA, KA, AAR and AAO contributed equally to this article. SA, AA and AAO_conception, proposal, ethical approval, recruitment, data analysis, and manuscript preparation. Data collection was done by NA, AO, MA, AMA, MB, HA, MMA, SYA, DKP, MYA, AHA, MSA, SAA and HSA. All authors read and approved the final manuscript.

\section{Funding}

This research received no external funding.

\section{Availability of data and materials}

Data are available upon request, please contact author for data requests.

\section{Declarations}

Ethics approval and consent to participate

Ethical approval of the current study was obtained from King Fahad Hofuf Review Board [IRB KFHH No. (H-05-HS-065)]. As patient data were de-identified and this was a retrospective study, written consent was not required.
Consent for publication

All authors agreed to this publication.

\section{Conflicts of interests}

The authors have no conflicts of interest to declare.

\section{Author details}

${ }^{1}$ Administration of Pharmaceutical Care, Alahsa Health Cluster, Ministry of Health, Rashdiah Street, P. O. Box 12944, Alahsa 31982, Saudi Arabia. ${ }^{2}$ Research Center, Almoosa Specialist Hospital, Alahsa, Saudi Arabia. ${ }^{3}$ School of Nursing, Wollongong University, Wollongong, Australia. ${ }^{4}$ College of Nursing, Princess NorahBint Abdul Rahman University, Riyadh, Saudi Arabia. ${ }^{5}$ Division of Allergy and Immunology, College of Medicine, King Faisal University, Alahsa, Saudi Arabia. ${ }^{6}$ Nursing Department, Al Jaber Hospital for Eye, Ear, Nose and Throat, Ministry of Health, Al-Hofuf, Saudi Arabia. ${ }^{7}$ General Surgery Department, Alomran General Hospital, Alahsa, Saudi Arabia. ${ }^{8}$ Internal Medicine Department, Alomran General Hospital, Alahsa, Saudi Arabia. ${ }^{9}$ Pharmacy Department, King Faisal General Hospital, Alahsa, Saudi Arabia. ${ }^{10}$ Infection Prevention and Control Department, Prince Saud Bin Jalawi Hospital, Alahsa, Saudi Arabia. ${ }^{11}$ Nursing Department, Maternity and Children Hospital, Alahsa, Saudi Arabia. ${ }^{12}$ Nursing Education Department, Maternity and Children Hospital, Alahsa, Saudi Arabia. ${ }^{13}$ Administration of Academic Affairs and Research, Ministry of Health, Alahsa, Saudi Arabia. ${ }^{14}$ Pharmacy Department, King Fahad Hofuf Hospital, Alahsa, Saudi Arabia. ${ }^{15}$ Planning and Research Department, Ministry of Health, Alahsa, Saudi Arabia. ${ }^{16}$ Histopathology Department, King Saud Medical City, Riyadh, Saudi Arabia. ${ }^{17}$ Intensive Care Unit Department, Dr. Sulaiman Al Habib Medical Group, Riyadh, Saudi Arabia. ${ }^{18}$ Molecular Diagnostics Laboratory, Johns Hopkins Aramco Healthcare, Dhahran, Saudi Arabia. ${ }^{19}$ Infectious Disease Unit, Specialty Internal Medicine, Johns Hopkins Aramco Healthcare, Dhahran, Saudi Arabia. ${ }^{20}$ Department of Medicine, Indiana University School of Medicine, Indianapolis, IN, USA. ${ }^{21}$ Department of Medicine, Johns Hopkins University School of Medicine, Baltimore, MD, USA. ${ }^{22}$ College of Medicine, Alfaisal University, Riyadh, Saudi Arabia. ${ }^{23}$ Research Center, Dr. Sulaiman Al Habib Medical Group, Riyadh, Saudi Arabia.

Received: 14 February 2021 Accepted: 10 May 2021

Published online: 24 May 2021

\section{References}

1. Chen N, Zhou M, Dong X, Qu J, Gong F, Han Y, Qiu Y, Wang J, Liu Y, Wei $Y$. Epidemiological and clinical characteristics of 99 cases of 2019 novel coronavirus pneumonia in Wuhan, China: a descriptive study. Lancet. 2020;395(10223):507-13.

2. World Health Organization. WHO Director-General's remarks at the media briefing on 2019-nCoV on 11 February 20202021 [cited 2 January 2021]. https://www.who.int/director-general/speeches/detail/who-direc tor-general-s-remarks-at-the-media-briefing-on-2019-ncov-on-11-febru ary-2020.

3. Rabaan AA, Al-Ahmed SH, Al-Malkey MK, Alsubki RA, Ezzikouri S, AlHababi FH, Sah R, Al Mutair A, Alhumaid S, Al-Tawfiq JA. Airborne transmission of SARS-CoV-2 is the dominant route of transmission: droplets and aerosols. Infez Med. 2021;29:10-9.

4. Saudi Ministry of Health. Novel Corona Virus (2019-nCoV) infection guidelines. Novel Corona Virus (2019-nCoV) infection guidelines 2021 [2 January 2021]. https://www.moh.gov.sa/en/CCC/healthp/regulations/ Documents/Novel\%20Corona\%20Virus\%20Infection\%20Guidelines.pdf.

5. Saudi Ministry of Health. $\mathrm{MOH}$ reports first case of Coronavirus infection 2021 [cited 2 January 2021]. https://www.moh.gov.sa/en/Ministry/Media Center/News/Pages/News-2020-03-02-002.aspx.

6. Saudi Ministry of Health. COVID 19 Dashboard 20212021 [cited 2 January 2021]. https://covid19.moh.gov.sa/.

7. Young BE, Ong SWX, Kalimuddin S, Low JG, Tan SY, Loh J, Ng O-T, Marimuthu K, Ang LW, Mak TM. Epidemiologic features and clinical course of patients infected with SARS-CoV-2 in Singapore. JAMA. 2020;323(15):1488-94.

8. Wang D, Hu B, Hu C, Zhu F, Liu X, Zhang J, Wang B, Xiang H, Cheng Z, Xiong Y. Clinical characteristics of 138 hospitalized patients with 2019 novel coronavirus-infected pneumonia in Wuhan, China. JAMA 2020;323(11):1061-9. 
9. Huang C, Wang Y, Li X, Ren L, Zhao J, Hu Y, Zhang L, Fan G, Xu J, Gu X. Clinical features of patients infected with 2019 novel coronavirus in Wuhan, China. Lancet. 2020;395(10223):497-506.

10. Vahidy FS, Drews AL, Masud FN, Schwartz RL, Boom ML, Phillips RA. Characteristics and outcomes of COVID-19 patients during initial peak and resurgence in the Houston metropolitan area. JAMA. 2020;324(10):998-1000.

11. Arentz M, Yim E, Klaff L, Lokhandwala S, Riedo FX, Chong M, Lee M. Characteristics and outcomes of 21 critically ill patients with COVID-19 in Washington State. JAMA. 2020;323(16):1612-4.

12. Livingston E, Bucher K. Coronavirus disease 2019 (COVID-19) in Italy. JAMA. 2020;323(14):1335.

13. Grasselli G, Pesenti A, Cecconi M. Critical care utilization for the COVID-19 outbreak in Lombardy, Italy: early experience and forecast during an emergency response. JAMA. 2020;323(16):1545-6.

14. Wu Z, McGoogan JM. Characteristics of and important lessons from the coronavirus disease 2019 (COVID-19) outbreak in China: summary of a report of 72314 cases from the Chinese Center for Disease Control and Prevention. JAMA. 2020;323(13):1239-42.

15. Guan W-J, Ni Z-Y, Hu Y, Liang W-H, Ou C-Q, He J-X, Liu L, Shan H, Lei C-L, Hui DS. Clinical characteristics of coronavirus disease 2019 in China. N Engl J Med. 2020;382(18):1708-20.

16. Al Mutair A, Alhumaid S, Alhuqbani WN, Zaidi ARZ, Alkoraisi S, Al-Subaie MF, AlHindi AM, Abogosh AK, Alrasheed AK, Alsharafi AA. Clinical, epidemiological, and laboratory characteristics of mild-to-moderate COVID-19 patients in Saudi Arabia: an observational cohort study. Eur J Med Res. 2020;25(1):1-8

17. Al-Omari A, Alhuqbani WN, Zaidi ARZ, Al-Subaie MF, AlHindi AM, Abogosh AK, Alrasheed AK, Alsharafi AA, Alhuqbani MN, Salih S. Clinical characteristics of non-intensive care unit COVID-19 patients in Saudi Arabia: a descriptive cross-sectional study. J Infect Public Health. 2020;13(11):1639-44.

18. World Health Organization. Clinical management of severe acute respiratory infection when novel coronavirus (2019-nCoV) infection is suspected 2020 [cited 30 April 2021]. https://apps.who.int/iris/bitstream/handle/ 10665/330893/WHO-nCoV-Clinical-2020.3-eng.pdf?sequence=1\&isAll owed $=y$.

19. Grünebaum A, Chervenak FA, McCullough LB, Dudenhausen JW, Bornstein E, Mackowiak PA. How fever is defined in COVID-19 publications: a disturbing lack of precision. J Perinat Med. 2020. https://doi.org/10.1515/ jpm-2020-0546.

20. Saudi Ministry of Health. ICU Triage, Admission, and Discharge Criteria during the COVID 19 pandemic V2 2020 [cited 30 April 2021]. https:// www.moh.gov.sa/Ministry/MediaCenter/Publications/Documents/ICUCriteria-during.pdf.

21. Gemmati D, Bramanti B, Serino ML, Secchiero P, Zauli G, Tisato V. COVID19 and individual genetic susceptibility/receptivity: role of ACE1/ACE2 genes, immunity, inflammation and coagulation. Might the double $\mathrm{X}$-chromosome in females be protective against SARS-CoV-2 compared to the single X-chromosome in males? Int J Mol Sci. 2020;21(10):3474.

22. Gadi N, Wu SC, Spihlman AP, Moulton VR. What's sex got to do with COVID-19? Gender-based differences in the host immune response to Coronaviruses. Front Immunol. 2020:11:2147.

23. Stokes EK, Zambrano LD, Anderson KN, Marder EP, Raz KM, Felix SEB, Tie Y, Fullerton KE. Coronavirus disease 2019 case surveillance-United States, January 22-May 30, 2020. Morb Mortal Wkly Rep. 2020;69(24):759.

24. Zhao Z, Chen A, Hou W, Graham JM, Li H, Richman PS, Thode HC, Singer AJ, Duong TQ. Prediction model and risk scores of ICU admission and mortality in COVID-19. PLoS ONE. 2020;15(7):e0236618.

25. Grasselli G, Zangrillo A, Zanella A, Antonelli M, Cabrini L, Castelli A, Cereda D, Coluccello A, Foti G, Fumagalli R. Baseline characteristics and outcomes of 1591 patients infected with SARS-CoV-2 admitted to ICUs of the Lombardy Region, Italy. JAMA. 2020;323(16):1574-81.

26. Schurz H, Salie M, Tromp G, Hoal EG, Kinnear CJ, Möller M. The X chromosome and sex-specific effects in infectious disease susceptibility. Hum Genomics. 2019;13(1):2.

27. Redondo-Sendino Á, Guallar-Castillón P, Banegas JR, Rodríguez-Artalejo F. Gender differences in the utilization of health-care services among the older adult population of Spain. BMC Public Health. 2006;6(1):1-9.
28. Sharma G, Volgman AS, Michos ED. Sex differences in mortality from COVID-19 pandemic: are men vulnerable and women protected? Case Rep. 2020;2(9):1407-10.

29. Johnson HD, Sholcosky D, Gabello K, Ragni R, Ogonosky N. Sex differences in public restroom handwashing behavior associated with visual behavior prompts. Percept Mot Skills. 2003;97(3):805-10.

30. De Vito A, Fiore V, Princic E, Geremia N, Panu Napodano CM, Muredda AA, Maida I, Madeddu G, Babudieri S. Predictors of infection, symptoms development, and mortality in people with SARS-CoV-2 living in retirement nursing homes. PLOS ONE. 2021;16(3):e0248009.

31. Khamis F, Al-Zakwani I, Al Naamani H, Al Lawati S, Pandak N, Omar MB, Al Bahrani M, Bulushi ZA, Al Khalili H, Al SI. Clinical characteristics and outcomes of the first 63 adult patients hospitalized with COVID-19: an experience from Oman. J Infect Public Health. 2020;13(7):906-13.

32. Alshukry A, Ali H, Ali Y, Al-Taweel T, Abu-Farha M, AbuBaker J, Devarajan S, Dashti AA, Bandar A, Taleb H. Clinical characteristics of Coronavirus Disease 2019 (COVID-19) patients in Kuwait. PLoS ONE. 2020;15(11):e0242768.

33. Wu C, Chen X, Cai Y, Zhou X, Xu S, Huang H, Zhang L, Zhou X, Du C, Zhang $Y$. Risk factors associated with acute respiratory distress syndrome and death in patients with coronavirus disease 2019 pneumonia in Wuhan, China. JAMA Intern Med. 2020;180(7):934-43.

34. De Vito A, Geremia N, Fiore V, Princic E, Babudieri S, Madeddu G. Clinical features, laboratory findings and predictors of death in hospitalized patients with COVID-19 in Sardinia, Italy. Eur Rev Med Pharmacol Sci. 2020;24(14):7861-8.

35. Abate SM, Ahmed Ali S, Mantfardo B, Basu B. Rate of Intensive Care Unit admission and outcomes among patients with coronavirus: a systematic review and meta-analysis. PLoS ONE. 2020;15(7):e0235653.

36. Patanavanich R, Glantz SA. Smoking is associated with COVID-19 progression: a meta-analysis. Nicotine Tob Res. 2020;22(9):1653-6.

37. Bao C, Liu X, Zhang H, Li Y, Liu J. Coronavirus disease 2019 (COVID-19) CT findings: a systematic review and meta-analysis. J Am Coll Radiol. 2020;17(6):701

38. Salehi S, Abedi A, Balakrishnan S, Gholamrezanezhad A, Coronavirus disease, (COVID-19): a systematic review of imaging findings in 919 patients. Am J Roentgenol. 2019;2020:1-7.

39. American College of Radiology. ACR recommendations for the use of chest radiography and computed tomography (CT) for suspected COVID-19 infection 2021 [2 January 2021]. https://www.acr.org/Advoc acy-and-Economics/ACR-Position-Statements/Recommendations-forChest-Radiography-and-CT-for-Suspected-COVID19-Infection.

40. Han X, Cao Y, Jiang N, Chen Y, Alwalid O, Zhang X, Gu J, Dai M, Liu J, Zhu W. Novel coronavirus disease 2019 (COVID-19) pneumonia progression course in 17 discharged patients: comparison of clinical and thin-section computed tomography features during recovery. Clin Infect Dis. 2020:71(15):723-31.

41. BMJ Best Practice. Coronavirus disease 2019 (COVID-19). Differentials 2021 [2 January 2021]. https://bestpractice.bmj.com/topics/en-gb/ 3000201/differentials.

42. Cohen PA, Hall L, John JN, Rapoport AB. The early natural history of SARS-CoV-2 infection: clinical observations from an urban, ambulatory COVID-19 clinic. Mayo Clin Proc. 2020;95(6):1124-6. https://doi.org/10. 1016/j.mayocp.2020.04.010.

43. Yang $X, Y u Y, X u$ J, Shu $H$, Liu H, Wu Y, Zhang L, Yu Z, Fang M, Yu T. Clinical course and outcomes of critically ill patients with SARS-CoV-2 pneumonia in Wuhan, China: a single-centered, retrospective, observational study. Lancet Respir Med. 2020;8(5):475-81.

44. Cheng F-Y, Joshi H, Tandon P, Freeman R, Reich DL, Mazumdar M, KohliSeth R, Levin M, Timsina P, Kia A. Using machine learning to predict ICU transfer in hospitalized COVID-19 Patients. J Clin Med. 2020;9(6):1668.

45. Wang F, Yang Y, Dong K, Yan Y, Zhang S, Ren H, Yu X, Shi X. Clinical characteristics of 28 patients with diabetes and COVID-19 in Wuhan, China. Endocrine Pract. 2020;26(6):668-74.

46. Roncon L, Zuin M, Rigatelli G, Zuliani G. Diabetic patients with COVID-19 infection are at higher risk of ICU admission and poor short-term outcome. J Clin Virol. 2020;127:104354.

47. Yang J, Zheng Y, Gou X, Pu K, Chen Z, Guo Q, Ji R, Wang H, Wang Y, Zhou $Y$. Prevalence of comorbidities and its effects in patients infected with SARS-CoV-2: a systematic review and meta-analysis. Int J Infect Dis. 2020:94:91-5. 
48. Caussy C, Pattou F, Wallet F, Simon C, Chalopin S, Telliam C, Mathieu D, Subtil F, Frobert E, Alligier M. Prevalence of obesity among adult inpatients with COVID-19 in France. Lancet Diabetes Endocrinol. 2020;8(7):562-4.

49. Kalligeros M, Shehadeh F, Mylona EK, Benitez G, Beckwith CG, Chan PA, Mylonakis E. Association of obesity with disease severity among patients with coronavirus disease 2019. Obesity. 2020;28(7):1200-4.

50. Siepmann T, Sedghi A, Barlinn J, Mirow L, Wolz M, Gruenewald T, Helbig S, Schroettner P, Winzer S, von Bonin S. Association of history of cerebrovascular disease with severity of COVID-19. J Neurol. 2020:1-12.

51. Patel U, Malik P, Shah D, Patel A, Dhamoon M, Jani V. Pre-existing cerebrovascular disease and poor outcomes of COVID-19 hospitalized patients: a meta-analysis. J Neurol. 2020. https://doi.org/10.1007/ s00415-020-10141-w.

52. Heilbronner C, Berteloot L, Tremolieres P, Dupic L, de Saint BL, Lesage F, Odièvre MH, de Marcellus C, Fourgeaud J, de Montalembert M. Patients with sickle cell disease and suspected COVID-19 in a paediatric intensive care unit. Br J Haematol. 2020;190(1):e21-4.

53. Panepinto JA, Brandow A, Mucalo L, Yusuf F, Singh A, Taylor B, Woods K, Payne AB, Peacock G, Schieve LA. Coronavirus disease among persons with sickle cell disease, United States, March 20-May 21, 2020. Emerg Infect Dis. 2020;26(10):2473.

54. Arlet J-B, de Luna G, Khimoud D, Odièvre M-H, de Montalembert M, Joseph L, Chantalat-Auger C, Flamarion E, Bartolucci P, Lionnet F. Prognosis of patients with sickle cell disease and COVID-19: a French experience. Lancet Haematol. 2020;7(9):e632-4.

55. COVID TC, Team R. Severe Outcomes Among Patients with Coronavirus Disease 2019 (COVID-19)-United States, February 12-March 16, 2020. 2020.

56. Roberts MB, Izzy S, Tahir Z, Al Jarrah A, Fishman JA, El Khoury J. COVID-19 in solid organ transplant recipients: Dynamics of disease progression and inflammatory markers in ICU and non-ICU admitted patients. Transplant Infect Dis. 2020;22(5):e13407.

57. Chu Y, Li T, Fang Q, Wang X. Clinical features of critically ill patients with confirmed COVID-19. J Infect. 2020;81(1):147-78.

58. Ferguson J, Rosser Jl, Quintero O, Scott J, Subramanian A, Gumma M, Rogers A, Kappagoda S. Characteristics and outcomes of coronavirus disease patients under nonsurge conditions, Northern California, USA, March-April 2020. Emerg Infect Dis. 2020;26(8):1679.

59. Garcia PDW, Fumeaux T, Guerci P, Heuberger DM, Montomoli J, RocheCampo F, Schuepbach RA, Hilty MP, Investigators R-I. Prognostic factors associated with mortality risk and disease progression in 639 critically ill patients with COVID-19 in Europe: Initial report of the international RISC-19-ICU prospective observational cohort. EClinicalMedicine. 2020;25:100449.

60. Vaira LA, Deiana G, Fois AG, Pirina P, Madeddu G, De Vito A, Babudieri S, Petrocelli M, Serra A, Bussu F. Objective evaluation of anosmia and ageusia in COVID-19 patients: single-center experience on 72 cases. Head Neck. 2020;42(6):1252-8.

61. Vaira LA, Hopkins C, Salzano G, Petrocelli M, Melis A, Cucurullo M, Ferrari M, Gagliardini L, Pipolo C, Deiana G. Olfactory and gustatory function impairment in COVID-19 patients: Italian objective multicenter-study. Head Neck. 2020;42(7):1560-9.
62. Callejon-Leblic MA, Moreno-Luna R, Del Cuvillo A, Reyes-Tejero IM, GarciaVillaran MA, Santos-Peña M, Maza-Solano JM, Martín-Jimenez DI, PalaciosGarcia JM, Fernandez-Velez C. Loss of smell and taste can accurately predict COVID-19 infection: a machine-learning approach. J Clin Med. 2021;10(4):570.

63. Tong JY, Wong A, Zhu D, Fastenberg JH, Tham T. The prevalence of olfactory and gustatory dysfunction in COVID-19 patients: a systematic review and meta-analysis. Otolaryngol-Head Neck Surg. 2020;163(1):3-11.

64. Vaira LA, Salzano G, De Riu G. The importance of olfactory and gustatory disorders as early symptoms of coronavirus disease (COVID-19). Br J Oral Maxillofac Surg. 2020;58(5):615-6.

65. Infectious Diseases Society of America. Guidelines on the Treatment and Management of Patients with COVID-19 2021 [cited 2 January 2021]. https://www.idsociety.org/practice-guideline/covid-19-guideline-treat ment-and-management/.

66. National Institutes of Health. Coronavirus Disease 2019 (COVID-19) Treatment Guidelines 2021 [cited 2 January 2021]. https://www.covid19tre atmentguidelines.nih.gov/.

67. Chan JF-W, Yao Y, Yeung M-L, Deng W, Bao L, Jia L, Li F, Xiao C, Gao H, Yu P. Treatment with lopinavir/ritonavir or interferon- $\beta 1 \mathrm{~b}$ improves outcome of MERS-CoV infection in a nonhuman primate model of common marmoset. J Infect Dis. 2015;212(12):1904-13.

68. Alhumaid S, Tobaiqy M, Albagshi M, Alrubaya A, Algharib F, Aldera A, Alali J. MERS-CoV transmitted from animal-to-human vs MERSCoV transmitted from human-to-human: comparison of virulence and therapeutic outcomes in a Saudi hospital. Trop J Pharm Res. 2018;17(6):1155-64.

69. Consortium WST. Repurposed antiviral drugs for COVID-19-Interim WHO SOLIDARITY trial results. New England Journal of Medicine. 2020.

70. Horby PW, Mafham M, Bell JL, Linsell L, Staplin N, Emberson J, Palfreeman A, Raw J, Elmahi E, Prudon B. Lopinavir-ritonavir in patients admitted to hospital with COVID-19 (RECOVERY): a randomised, controlled, openlabel, platform trial. Lancet. 2020;396(10259):1345-52.

71. Alhumaid S, Mutair AA, Alawi ZA, Alhmeed N, Zaidi ARZ, Tobaiqy M. Efficacy and safety of lopinavir/ritonavir for treatment of COVID-19: a systematic review and meta-analysis. Trop Med Infect Dis. 2020;5(4):180

72. Rabaan AA, Al-Ahmed SH, Muhammad J, Khan A, Sule AA, Tirupathi R, Mutair AA, Alhumaid S, Al-Omari A, Dhawan M. Role of inflammatory cytokines in COVID-19 patients: A review on molecular mechanisms, immune functions, immunopathology and immunomodulatory drugs to counter cytokine storm. Vaccines. 2021;9(5):436.

73. Kim PS, Read SW, Fauci AS. Therapy for early COVID-19: a critical need. JAMA. 2020;324(21):2149-50.

\section{Publisher's Note}

Springer Nature remains neutral with regard to jurisdictional claims in published maps and institutional affiliations.

Ready to submit your research? Choose BMC and benefit from

- fast, convenient online submission

- thorough peer review by experienced researchers in your field

- rapid publication on acceptance

- support for research data, including large and complex data types

- gold Open Access which fosters wider collaboration and increased citations

- maximum visibility for your research: over 100M website views per year

At BMC, research is always in progress.

Learn more biomedcentral.com/submissions 